\title{
Sigma-omega meson coupling and properties of nuclei and nuclear matter
}

\author{
Maryam M. Haidari and Madan M. Sharma* \\ Physics Department, Kuwait University, Kuwait 13060
}

\begin{abstract}
We have constructed a Lagrangian model with a coupling of $\sigma$ and $\omega$ mesons in the relativistic mean-field theory. Properties of finite nuclei and nuclear matter are explored with the new Lagrangian model SIG-OM. The study shows that an excellent description of binding energies and charge radii of nuclei over a large range of isospin is achieved with SIG-OM. With an incompressibility of nuclear matter $K=265 \mathrm{MeV}$, it is also able to describe the breathing-mode isoscalar giant monopole resonance energies appropriately. It is shown that the high-density behaviour of the equation of state of nuclear and neutron matter with the $\sigma-\omega$ coupling is much softer than that of the non-linear scalar coupling model.
\end{abstract}

Key words: Relativistic mean-field, effective Lagrangian approach, $\sigma-\omega$ coupling, finite nuclei, ground-state properties, $\mathrm{Sn}$ and $\mathrm{Pb}$ isotopes, breathing-mode giant monopole resonance, incompressibility, equation of state, nuclear matter, neutron matter.

PACS: 21.30.Fe, 21.10.Dr, 21.60.-n, 24.10.Cn, 24.10.Jv, 21.65.+f

\section{Introduction}

The relativistic mean-field (RMF) theory [1,2,3,4] has been a successful approach to describing properties of nuclei along the stability line as well as far away from it [5,6,7]. Due to the Dirac-Lorentz structure of spin-orbit interaction, it has been shown to be advantageous over the conventional nonrelativistic Skyrme theory in describing properties such as anomalous isotope

\footnotetext{
* Corresponding author

Email address: sharma@kuc01.kuniv.edu.kw (Madan M. Sharma).
} 
shifts in $\mathrm{Pb}$ nuclei [8]. It was shown [9] that an isospin dependence of the spinorbit interaction or essentially a lack of it is responsible for the anomalous behaviour of the isotope shifts. The non-relativistic approaches based upon the Skyrme and Gogny forces have been unable to reproduce the kink [10]. On the other hand, by significant alterations in the isospin dependence of the spin-orbit potential in the Skyrme theory [11, it becomes possible to describe the above anomaly in isotope shifts.

The prevalent model within the RMF theory is that of non-linear couplings of $\sigma$ meson [12]. Herein, the nonlinear scalar self-couplings of $\sigma$ meson play a pivotal role in describing finite nuclei. It was realized earlier on [12] that $\sigma^{3}+$ $\sigma^{4}$ terms have ingredients appropriate for a proper description of the surface properties of nuclei. By virtue of this, the scalar self-coupling terms have become indispensable for finite nuclei. One of the first nuclear forces constructed for calculation of ground-state properties of finite nuclei was NL1 [2]. A larger value of the asymmetry energy of NL1, however, made its application to nuclei far away from the stability line difficult. Within this model, one of the first successful nuclear forces was NL-SH [6] which was obtained with a significant improvement in the asymmetry energy. The force NL-SH has an incompressibility of nuclear matter which is on the higher side of physical acceptable region. Sunsequently, force NL3 was obtained [7] with an improvement in the nuclear incompressibility of nuclear matter. This has in meanwhile become successful in several respects.

A problem that afflicts the model with $\sigma^{3}+\sigma^{4}$, in general, is that it gives an equation of state (EOS) of nuclear and neutron matter that is very stiff. Consequently, it is considered as incompatible with the observed spectrum of neutron star masses. A quartic term $\omega^{4}$ was added recently to the above model that was intended to improve the shell effects in nuclei [13]. It has subsequently been shown that the addition of the vector self-coupling of $\omega$ meson has the advantage of softening the EOS of nuclear matter [14]15].

Variations in the relativistic approach based upon point-coupling model [16]17]18] or of density-dependent meson couplings [19,20,21,22,23] have been made. With the inclusion of a larger number of parameters a good degree of success has been achieved [22]. There has also been significant discussion on the use of the effective field theoretical approach based upon expansion of the interaction Lagrangian in higher order terms in fields [24]25|26]. Addition of higher order terms and cross terms between the fields are shown to improve description of properties of finite nuclei [25]. Recently, a coupling between $\sigma$ and $\rho$ meson has shown to be promising in reduction of neutron skin to reasonably accepted values as well as for obtaining a softer EOS of nuclear matter [27]. Thus, within the effective Lagrangian approach, the field of the RMF theory has become widely open with many possibilities of interaction terms. It is, however, not known as to what form of density dependence of nucleon-meson 
interactions or of meson-meson interactions would be the most suitable one. In other words, which terms nature prefers in the expansion scheme is not known a priori. Emergence of nuclear data in extreme regions of the periodic table including those on finite nuclei, giant resonances and neutron star masses put nuclear interactions to test.

With the initial success of the RMF theory in nuclear structure, there is thus a strong need to construct a theory that should be appropriate for broader aspects of finite nuclei as well as for nuclear matter. As improved and refined predictions of nuclear properties especially the masses (binding energies) of nuclei are required for regions often very far away from the stability line such as those for r-process nucleosynthesis, it is pertinent to devise new models and approaches to deliver the same. This may include other degrees of freedom or alternative forms of interactions.

In this work, we have explored the RMF Lagrangian in the effective Lagrangian approach with a limited number of terms in the expansion with a view to see as to whether such an approach is feasible. We have restricted ourselves to a meson-meson interaction term between $\sigma$ and $\omega$ mesons. Specifically, we have added a coupling of the form $\sigma^{2} \omega^{2}$, in addition to the $\sigma^{3}+\sigma^{4}$ couplings which have so far been the most important terms in the RMF Lagrangian and are indispensable for finite nuclei and nuclear matter.

\section{Relativistic Mean-Field Theory: Formalism}

The basic (linear) RMF Lagrangian that describes nucleons as Dirac spinors interacting with the meson fields is given by [1]

$$
\begin{aligned}
\mathcal{L}_{0}= & \bar{\psi}\left(\not p-g_{\omega} \psi-g_{\rho} \vec{\phi} \vec{\tau}-\frac{1}{2} e\left(1-\tau_{3}\right) A-g_{\sigma} \sigma-M_{N}\right) \psi \\
& +\frac{1}{2} \partial_{\mu} \sigma \partial^{\mu} \sigma-\frac{1}{2} m_{\sigma}^{2} \sigma^{2}-\frac{1}{4} \Omega_{\mu \nu} \Omega^{\mu \nu}+\frac{1}{2} m_{\omega}^{2} \omega_{\mu} \omega^{\mu} \\
& -\frac{1}{4} \vec{R}_{\mu \nu} \vec{R}^{\mu \nu}+\frac{1}{2} m_{\rho}^{2} \vec{\rho}_{\mu} \vec{\rho}^{\mu}-\frac{1}{4} F_{\mu \nu} F^{\mu \nu}
\end{aligned}
$$

where $M_{N}$ is the bare nucleon mass and $\psi$ is its Dirac spinor. Nucleons interact with $\sigma, \omega$, and $\rho$ mesons, where $g_{\sigma}, g_{\omega}$, and $g_{\rho}$ are the respective coupling constants. The photonic field is represented by the electromagnetic vector $A^{\mu}$.

The nonlinear $\sigma$-meson-couplings added by Boguta and Bodmer [12] are of the form 


$$
U_{N L}=\frac{1}{3} g_{2} \sigma^{3}+\frac{1}{4} g_{3} \sigma^{4}
$$

The parameters $g_{2}$ and $g_{3}$ are the nonlinear couplings of $\sigma$-meson in the conventional $\sigma^{3}+\sigma^{4}$ model. The effective Lagrangian that is commonly used is

$$
\mathcal{L}_{\text {eff }}=\mathcal{L}_{0}+U_{N L}
$$

Here, we add an interaction term between the $\sigma$ and $\omega$ meson. Then, the effective Lagrangian becomes

$$
\mathcal{L}_{e f f}=\mathcal{L}_{0}+U_{N L}+\frac{1}{2} g_{\sigma \omega} \sigma^{2} \omega_{\mu} \omega^{\mu}
$$

The last term represents the coupling between $\sigma$ and $\omega$ meson that we introduce. The constant $g_{\sigma \omega}$ is the coupling constant of $\sigma$ and $\omega$ meson-meson interaction. The field tensors of the vector mesons and of the electromagnetic field take the following form:

$$
\begin{aligned}
& \Omega^{\mu \nu}=\partial^{\mu} \omega^{\nu}-\partial^{\nu} \omega^{\mu} \\
& \mathbf{R}^{\mu \nu}=\partial^{\mu} \boldsymbol{\rho}^{\nu}-\partial^{\nu} \boldsymbol{\rho}^{\mu} \\
& F^{\mu \nu}=\partial^{\mu} \mathbf{A}^{\nu}-\partial^{\nu} \mathbf{A}^{\mu} .
\end{aligned}
$$

The variational principle gives rise to the Dirac equation:

$$
\{-i \alpha \cdot \nabla+V(\mathbf{r})+\beta m *\} \psi_{i}=\epsilon_{i} \psi_{i},
$$

where $V(\mathbf{r})$ represents the vector potential:

$$
V(\mathbf{r})=g_{\omega} \omega_{0}(\mathbf{r})+g_{\rho} \tau_{3} \rho_{0}(\mathbf{r})+e \frac{1-\tau_{3}}{2} A_{0}(\mathbf{r}),
$$

and $S(\mathbf{r})$ is the scalar potential

$$
S(\mathbf{r})=g_{\sigma} \sigma(\mathbf{r})
$$

which defines the effective mass as given by

$$
m^{*}(\mathbf{r})=m+S(\mathbf{r})
$$

The corresponding Klein-Gordon equations can be written as 


$$
\begin{aligned}
\left(-\Delta+m_{\sigma}^{* 2}\right) \sigma & =-g_{\sigma} \bar{\psi} \psi \\
\left(-\Delta+m_{\omega}^{* 2}\right) \omega_{\nu} & =g_{\omega} \bar{\psi} \gamma_{\nu} \psi \\
\left(-\Delta+m_{\rho}^{2}\right) \vec{\rho}_{\nu} & =g_{\rho} \bar{\psi} \gamma_{\nu} \vec{\tau} \psi \\
-\Delta A_{\nu} & =\frac{1}{2} e \bar{\psi}\left(1+\tau_{3}\right) \gamma_{\nu} \psi,
\end{aligned}
$$

where,

$$
\begin{aligned}
& m_{\sigma}^{* 2}=m_{\sigma}^{2}+g_{2} \sigma+g_{3} \sigma^{2}-g_{\sigma \omega} \omega_{0}^{2} \\
& m_{\omega}^{* 2}=m_{\omega}^{2}+g_{\sigma \omega} \sigma^{2} .
\end{aligned}
$$

These equations imply an implicit density dependence of $\sigma$ and $\omega$ meson masses. These density dependences are then responsible for the density dependence of nuclear interaction in a nucleus. This is in contrast to the nonrelativistic Skyrme approach wherein the density functional is well-defined at the outset. An explicit density dependence of mesons has been introduced in refs. [20,22,23]. This approach requires several additional parameters besides the usual coupling constants in order to model a density dependence whose form is not known a priori.

For the case of an even-even nucleus with a time-reversal symmetry, the spatial components of the vector fields $\boldsymbol{\omega}, \boldsymbol{\rho}_{\mathbf{3}}$ and A vanish. The Klein-Gordon equations for the meson fields are then time-independent inhomogeneous equations with the nucleon densities as sources:

$$
\begin{aligned}
& \left(-\Delta+m_{\sigma}^{2}\right) \sigma(\mathbf{r})=-g_{\sigma} \rho_{s}(\mathbf{r})-g_{2} \sigma^{2}(\mathbf{r})-g_{3} \sigma^{3}(\mathbf{r})+g_{\sigma \omega} \sigma(\mathbf{r}) \omega_{0}^{2}(\mathbf{r}) \\
& \left(-\Delta+m_{\omega}^{2}\right) \omega_{0}(\mathbf{r})=g_{\omega} \rho_{v}(\mathbf{r})-g_{\sigma \omega} \sigma^{2}(\mathbf{r}) \omega_{0}(\mathbf{r}) \\
& \left(-\Delta+m_{\rho}^{2}\right) \rho_{0}(\mathbf{r})=g_{\rho} \rho_{3}(\mathbf{r}) \\
& -\Delta A_{0}(\mathbf{r})=e \rho_{c}(\mathbf{r}) .
\end{aligned}
$$

For the mean-field, the nucleon spinors provide the corresponding source terms:

$$
\begin{aligned}
\rho_{s} & =\sum_{i=1}^{A} \bar{\psi}_{i} \psi_{i} \\
\rho_{v} & =\sum_{i=1}^{A} \psi_{i}^{+} \psi_{i} \\
\rho_{3} & =\sum_{p=1}^{Z} \psi_{p}^{+} \psi_{p}-\sum_{n=1}^{N} \psi_{n}^{+} \psi_{n} \\
\rho_{c} & =\sum_{p=1}^{Z} \psi_{p}^{+} \psi_{p},
\end{aligned}
$$

where the sums are taken over the valence nucleons only. 
The stationary state solutions $\psi_{i}$ are obtained from the coupled system of Dirac and Klein-Gordon equations self-consistently. The solution of the Dirac equation is obtained by using the method of oscillator expansion [5]. The ground-state of the nucleus is described by a Slater determinant $\mid \Phi>$ of single-particle spinors $\psi_{i}(\mathrm{i}=1,2, \ldots . \mathrm{A})$.

The centre-of-mass correction is added using the harmonic oscillator estimate:

$$
E_{\text {c.m. }}=\frac{3}{4} .41 A^{-1 / 3} .
$$

\section{The Lagrangian Parameter set}

The parameters of the new Lagrangian model are obtained by fitting binding energies and charge radii of a set of nuclei within the RMF theory. The procedure has been described in ref. 66. The nuclei included are ${ }^{16} \mathrm{O},{ }^{40} \mathrm{Ca}$, ${ }^{48} \mathrm{Ca},{ }^{90} \mathrm{Zr},{ }^{116} \mathrm{Sn},{ }^{124} \mathrm{Sn},{ }^{132} \mathrm{Sn}$, and ${ }^{208} \mathrm{~Pb}$. The Sn isotopes ${ }^{116} \mathrm{Sn},{ }^{124} \mathrm{Sn}$ and ${ }^{132} \mathrm{Sn}$ are included with a view to take into account the broad range of isospin dependence of the nuclear interaction. For open-shell nuclei, the pairing is included within the BCS scheme, where the pairing gaps are obtained from the experimental masses of neighbouring nuclei.

The binding energies and charge radii of nuclei have been used as constraints. In addition, we have tagged the spin-orbit splitting of $p_{3 / 2}-p_{1 / 2}$ in ${ }^{16} \mathrm{O}$. However, we have avoided putting explicit conditions or constraints on the nuclear matter properties. The $\omega$ and $\rho$ meson masses have been fixed at their empirical values. The procedure of obtaining the parameter sets of the new Lagrangian is the same as that applied in obtaining other forces such as NL-SH and NL3. It may recalled that the set NL3 was obtained by putting constraints explicitly on the nuclear matter properties as well.

The parameters of the Lagrangian thus obtained (here named as SIG-OM) are listed in Table 1. These are compared with those of the forces NL-SH and NL3 with the nonlinear scalar self-couplings. The coupling constant $g_{3}$ with SIG-OM is positive in contrast with that of NL-SH and NL3, where it is predominantly negative. It has been argued [2] that a negative $g_{3}$ has a consequence in that the spectrum of the full theory is not bound from below and that renormalization of the scalar field is not possible.

Solving the equations for nuclear matter at the saturation point, equilibrium properties of the nuclear matter are calculated. Nuclear properties arising from the parameters of SIG-OM are shown in Table 2. A comparison is made with the properties of NL-SH and NL3. The saturation density and binding energy per nucleon for SIG-OM are very close to those of NL-SH and NL3 and are in 
Table 1

The parameters of the Lagrangian SIG-OM with the coupling between $\sigma$ and $\omega$ mesons. The Lagrangian sets with the non-linear scalar self-coupling NL-SH, NL3 are shown for comparison.

\begin{tabular}{llll}
\hline Parameters & SIG-OM & NL-SH & NL3 \\
\hline $\mathrm{M}(\mathrm{MeV})$ & 939.0 & 939.0 & 939.0 \\
$m_{\sigma}(\mathrm{MeV})$ & 505.9263 & 526.0592 & 508.1941 \\
$m_{\omega}(\mathrm{MeV})$ & 783.0 & 783.0 & 782.501 \\
$m_{\rho}(\mathrm{MeV})$ & 763.0 & 763.0 & 763.0 \\
$g_{\sigma}$ & 10.0429 & 10.4436 & 10.2169 \\
$g_{\omega}$ & 12.7668 & 12.9451 & 12.8675 \\
$g_{\rho}$ & 4.4752 & 4.3828 & 4.4744 \\
$g_{2}\left(\mathrm{fm}^{-1}\right)$ & -7.9223 & -6.9099 & -10.4307 \\
$g_{3}$ & 12.4601 & -15.8337 & -28.8851 \\
$g_{\sigma \omega}$ & 35.6922 & 0.0 & 0.0 \\
\hline
\end{tabular}

Table 2

The nuclear matter (NM) properties due to the force SIG-OM. Properties of the sets NL-SH and NL3 are also given for comparison.

\begin{tabular}{lccc}
\hline NM Properties & SIG-OM & NL-SH & NL3 \\
\hline$\rho_{0}\left(\mathrm{fm}^{-3}\right)$ & 0.149 & 0.146 & 0.148 \\
$a_{v}(\mathrm{MeV})$ & -16.30 & -16.33 & -16.24 \\
$K(\mathrm{MeV})$ & 265.2 & 354.9 & 271.6 \\
$m^{*}$ & 0.622 & 0.597 & 0.595 \\
$a_{4}(\mathrm{MeV})$ & 37.0 & 36.1 & 37.4 \\
\hline
\end{tabular}

physically acceptable region. The incompressibility of nuclear matter $K$ that arises from SIG-OM is $265.2 \mathrm{MeV}$. It is slightly smaller than that of NL3 $(K=271.6 \mathrm{MeV})$. The effective mass at the saturation point with SIG-OM is $m *=0.62$. It is slightly bigger than a value of $\sim 0.60$ for NL-SH and NL3. The asymmetry energy $a_{4}$ of SIG-OM is $37.0 \mathrm{MeV}$. It is comparable to that of NL-SH and NL3 and is, however, still bigger than the received empirical value of $\sim 33 \mathrm{MeV}$. 


\section{Results and discussion}

\subsection{Ground-state properties of spherical nuclei}

The binding energies and charge radii of nuclei calculated with SIG-OM in the RMF theory are presented in Tables 3. Results with NL-SH and NL3 are also given for comparison. Table 3 shows the binding energies achieved for the key nuclei included in the fit along with those of several other isotopes of Sn and $\mathrm{Pb}$. The experimental binding energies [28] and charge radii where available are shown in the last column. The binding energies of the nuclei included in the fit are reproduced well by SIG-OM with a slight overbinding for ${ }^{16} \mathrm{O}$. The set includes three doubly magic nuclei of ${ }^{100} \mathrm{Sn},{ }^{132} \mathrm{Sn}$ and ${ }^{208} \mathrm{~Pb}$. It is important that any newly developed Lagrangian model should be able to describe the binding energies of closed-shell nuclei. Comparing the binding energy of ${ }^{132} \mathrm{Sn}$ and ${ }^{208} \mathrm{~Pb}$ due to $\mathrm{SIG}-\mathrm{OM}$ with the experimental data, one can see that there is a good agreement for ${ }^{132} \mathrm{Sn}$. For ${ }^{208} \mathrm{~Pb}$, SIG-OM overestimates the energy by $\sim 1.5 \mathrm{MeV}$. This is, however, much improved as compared to that of NL$\mathrm{SH}$ and NL3, where these sets overestimate the binding energy of ${ }^{208} \mathrm{~Pb}$ by $\sim 3-4 \mathrm{MeV}$. The binding energies of other isotopes of $\mathrm{Sn}$ and $\mathrm{Pb}$ nuclei obtained with SIG-OM show an excellent agreement with the experimental data as compared to NL-SH and NL3.

The case of ${ }^{100} \mathrm{Sn}$ needs a special mention. Almost all Lagrangian sets in use in the RMF theory overestimate the binding energy of ${ }^{100} \mathrm{Sn}$ significantly. This includes both NL-SH and NL3 which overestimate the binding energy by $\sim 4-5 \mathrm{MeV}$. The prediction of SIG-OM for ${ }^{100} \mathrm{Sn}$ is surprisingly close to the experimental value. This has, however, not affected (underpushed) the binding

energies of ${ }^{116} \mathrm{Sn},{ }^{124} \mathrm{Sn}$ and ${ }^{132} \mathrm{Sn}$ in the isotopic chain of $\mathrm{Sn}$. Thus, SIG-OM provides the best available description of doubly magic nucleus ${ }^{100} \mathrm{Sn}$.

The charge radii of nuclei calculated with SIG-OM are given in parentheses in Table 3. The SIG-OM values describes the experimental charge radii well. This is especially the case for $\mathrm{Pb}$ isotopes where SIG-OM values are closer to the experimental data. In comparison, NL3 overestimates the experimental charge radii of $\mathrm{Pb}$ isotopes. For the $\mathrm{Sn}$ isotopes, NL3 values are, however, closer to the experimental data. In comparison, charge radii for heavier isotopes with NL-SH are in close proximity to the experimental data. With the new Lagrangian model SIG-OM, there is a general agreement of its charge radii with the experimental data especially for heavier nuclei. 
Table 3

The binding energies in $\mathrm{MeV}$ and charge radii (parentheses) in fm of spherical nuclei obtained with SIG-OM. The values for NL3 and NL-SH are also shown for comparison. The empirical values (exp.) are shown in the last column

\begin{tabular}{lcccc}
\hline Nucleus & SIG-OM & NL-SH & NL3 & exp. \\
\hline${ }^{16} \mathrm{O}$ & $-129.2(2.699)$ & $-128.4(2.699)$ & $-128.8(2.728)$ & $-127.6(2.730)$ \\
${ }^{40} \mathrm{Ca}$ & $-343.3(3.440)$ & $-340.1(3.452)$ & $-342.0(3.469)$ & $-342.1(3.450)$ \\
${ }^{48} \mathrm{Ca}$ & $-414.6(3.454)$ & $-415.0(3.462)$ & $-415.1(3.471)$ & $-416.0(3.451)$ \\
${ }^{76} \mathrm{Ni}$ & $-631.8(3.920)$ & $-634.4(3.920)$ & $-634.1(3.926)$ & -633.1 \\
${ }^{90} \mathrm{Zr}$ & $-783.1(4.282)$ & $-782.9(4.282)$ & $-782.6(4.287)$ & $-783.9(4.258)$ \\
${ }^{100} \mathrm{Sn}$ & $-826.0(4.465)$ & $-830.6(4.467)$ & $-829.2(4.473)$ & -824.5 \\
${ }^{116} \mathrm{Sn}$ & $-988.8(4.593)$ & $-987.9(4.599)$ & $-987.7(4.611)$ & $-988.7(4.626)$ \\
${ }^{124} \mathrm{Sn}$ & $-1049.9(4.645)$ & $-1050.1(4.651)$ & $-1050.2(4.661)$ & $-1050.0(4.673)$ \\
${ }^{132} \mathrm{Sn}$ & $-1102.9(4.698)$ & $-1105.9(4.702)$ & $-1105.4(4.708)$ & -1102.9 \\
${ }^{202} \mathrm{~Pb}$ & $-1591.8(5.480)$ & $-1596.0(5.480)$ & $-1592.6(5.497)$ & $-1592.2(5.473)$ \\
${ }^{208} \mathrm{~Pb}$ & $-1638.3(5.506)$ & $-1640.4(5.509)$ & $-1639.6(5.523)$ & $-1636.7(5.503)$ \\
${ }^{214} \mathrm{~Pb}$ & $-1663.8(5.563)$ & $-1664.3(5.562)$ & $-1661.6(5.581)$ & $-1663.3(5.558)$ \\
\hline
\end{tabular}

\subsection{The isotopic chain of Sn nuclei}

With the new Lagrangian model SIG-OM, we have performed a case study. The isotopic chain of Sn nuclei with the experimental masses available from the proton drip-line doubly-magic nucleus ${ }^{100} \mathrm{Sn}$ to another doubly-magic nucleus ${ }^{132} \mathrm{Sn}$ offers unique data for a complete coverage of the shell from $N=50$ to $N=82$. We have performed the Relativistic Hartree-Bogoliubov calculations for $\mathrm{Sn}$ isotopes. The details of the method are provided in ref. [13]. The Gogny force D1S has been used in the pairing channel. It has been shown that the Gogny force is able to represent the pairing properties of $\mathrm{Sn}, \mathrm{Pb}$ and other nuclei successfully [29].

The binding energies of Sn nuclei obtained with SIG-OM are shown in Fig. 1. A comparison is made with the predictions of NL3. Here, we have also included the results due to NL-SV1 [13]. The force NL-SV1 is based upon the model with the quartic coupling of $\omega$ meson. The results show a significant difference between the predictions of SIG-OM and both the other Lagrangian models in the regions near the closed shells. Whereas NL-SV1 shows a good agreement with the data in the region $106<A<132$, NL3 shows a reasonably good agreement with the data in the region $106<A<126$. Both NL3 and NL-SV1 


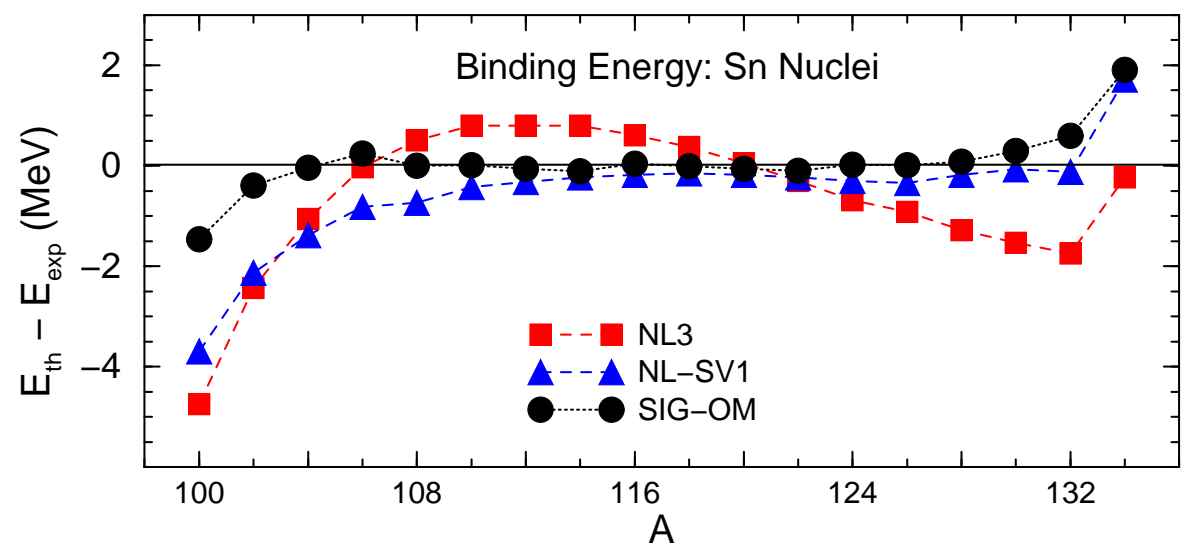

Fig. 1. The binding energies of $\mathrm{Sn}$ isotopes calculated using the Relativistic Hartree-Bogoliubov method with the Lagrangian SIG-OM. The results obtained with NL3 and NL-SV1 are also shown for comparison.

show significant deviations from the experimental values below $A<106$. For the double-magic nucleus ${ }^{100} \mathrm{Sn}$, the overbinding amounts to $\sim 4-5 \mathrm{MeV}$. This problem of overbinding of magic nuclei and by implication the presence of a strong shell energy is not limited to theoretical models alone. The phenomenon of arches at the magic numbers persists both in microscopic theories and mass formulae alike.

Viewing the binding energy curves in Fig. 1, one can see clearly that SIG-OM shows an excellent agreement with the experimental data all over the range of the shell. It shows a much smaller deviation from the data at $A=100$. The well-known arch-like pattern that is exhibited by NL-SV1 and NL3 is reduced significantly with SIG-OM. Thus, SIG-OM provides a very good description of the binding energies of the $\mathrm{Sn}$ isotopes over the whole region between the two magic numbers.

\subsection{Charge radii and isotopic shifts of $\mathrm{Pb}$ nuclei}

The salient feature of the $\mathrm{Pb}$ chain is the presence of a characteristic kink in charge radii and isotopic shifts at the magic number $N=82$. This behaviour of charge radii was considered for long to be anomalous [10]. Thus, the charge radii and isotopic shifts for $\mathrm{Pb}$ chain have become a standard test bench for any new model. In order to view as what response the new model SIG-OM gives for charge radii, we have performed $\mathrm{RMF}+\mathrm{BCS}$ calculations for the isotopes of $\mathrm{Pb}$. The charge radii of $\mathrm{Pb}$ isotopes calculated with $\mathrm{SIG-OM}$ are shown in Fig. 2. A comparison is made with the charge radii obtained with NL-SH and NL3. The experimental values [30] are shown for a comparison. A kink across 
$A=208(N=82)$ arises in all the curves. The SIG-OM values describe the experimental data as well as NL-SH does. Whereas both SIG-OM and NL-SH show some minor differences with the data for isotopes lighter than ${ }^{208} \mathrm{~Pb}$, for the heavier isotopes SIG-OM values show a better agreement with the data. The data point for ${ }^{208} \mathrm{~Pb}$ is significant as charge radius of this nucleus has been measured using various approaches and its value $\left(r_{c}=5.503 \mathrm{fm}\right)$ is known with a very high precision. The charge radius of ${ }^{208} \mathrm{~Pb}$ with SIG-OM is $5.506 \mathrm{fm}$. It is very close to the experimental value. The value with NL-SH is $5.509 \mathrm{fm}$ which is slightly larger than the experimental data. In comparison, the charge radius of ${ }^{208} \mathrm{~Pb}$ with NL3 is $5.523 \mathrm{fm}$. The results of Fig. 2 show that NL3 overestimates the charge radii of the $\mathrm{Pb}$ isotopes systematically.
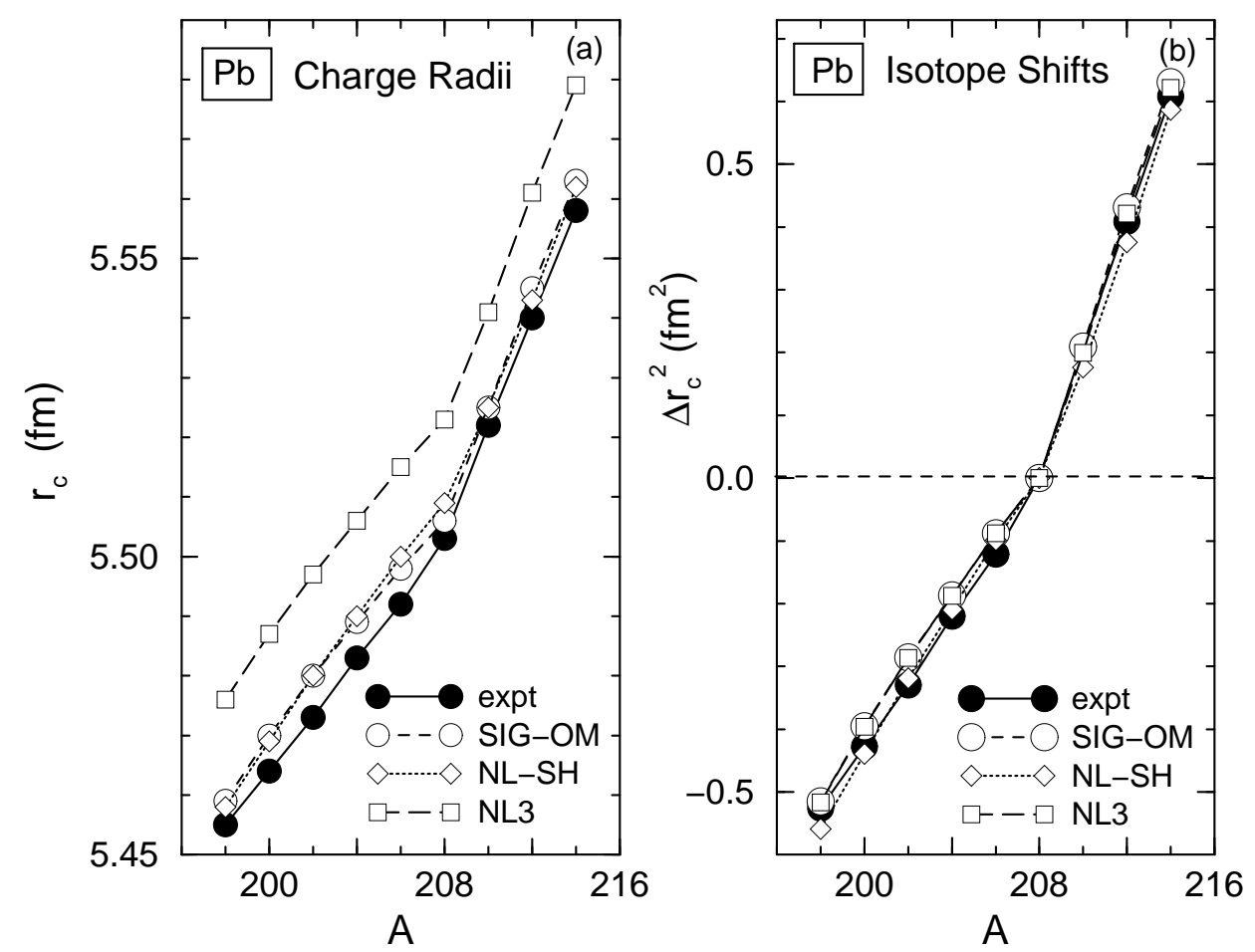

Fig. 2. The charge radii calculated with SIG-OM, NL-SH and NL3. Experimental values are also shown for comparison

The isotope shifts $\Delta r_{c}^{2}=r_{c}^{2}(A)-r_{c}^{2}(208)$ of $\mathrm{Pb}$ nuclei have been measured with a significant precision using laser-beam atomic spectroscopy [30]. Whilst ${ }^{208} \mathrm{~Pb}$ acts as a reference point in the measurement of $\Delta r_{c}^{2}$, the actual value of the charge radius of ${ }^{208} \mathrm{~Pb}$ does enter into the values of the isotopes shifts obtained from the measurements. We show in Fig. 2(b) the isotopes shifts calculated using the charge radii from SIG-OM, NL-SH and NL3. The experimental data [30] are shown directly. The theoretical values match the experimental data very well irrespective of the Lagrangian model used. Even the larger charge radii predicted by NL3 cancel out in the calculation of $\Delta r_{c}^{2}$. Apparently, the isotope shifts of SIG-OM are very close to those due to NL3. We can con- 
clude that the RMF theory describes the experimental isotope shifts very well irrespective of the nature of the Lagrangian model employed with barely perceptible differences amongst various forces.

\subsection{Nuclei away from the stability line}

Mass measurements with high precision on nuclei far away from the stability are being performed at various facilities and new experimental data are emerging currently. Such data provide a crucial test bench for various nuclear models in regions away from the stability line. In order to test the predictive power of the Lagrangian SIG-OM, we have performed deformed RMF calculations for some nuclei on which experimental data has been obtained recently. The total binding energy of some isotopes of $\mathrm{Si}, \mathrm{Sr}$, and Mo and a few others obtained with SIG-OM is shown in Table 5. A comparison is made with NL3. The experimental binding energies obtained from high-precision experimental masses on Si [31], Sr [32]) and Mo [33]) are used for comparison.

Table 4

The binding energy $\mathrm{E}$ (in $\mathrm{MeV}$ ) and quadrupole deformation $\beta_{2}$ of nuclei away from the stability line calculated with SIG-OM. The deviation of the binding energy from the experimental value, $\Delta \mathrm{E}=\mathrm{E}-\mathrm{E}_{\text {exp }}$, is also shown. A comparison is made with the predictions of NL3.

\begin{tabular}{ccccccc}
\hline Nucleus & \multicolumn{3}{c}{ SIG-OM } & \multicolumn{3}{c}{$\mathrm{NL3}$} \\
& $\mathrm{E}$ & $\beta_{2}$ & $\Delta E$ & $\mathrm{E}$ & $\beta_{2}$ & $\Delta E$ \\
\hline${ }^{36} \mathrm{Si}$ & -292.4 & 0.02 & -0.4 & -293.5 & 0.0 & -1.5 \\
${ }^{38} \mathrm{Si}$ & -300.0 & 0.28 & -0.2 & -301.3 & 0.27 & -1.5 \\
${ }^{40} \mathrm{Si}$ & -306.6 & 0.36 & -0.1 & -307.9 & 0.34 & -1.4 \\
${ }^{42} \mathrm{Si}$ & -313.3 & -0.35 & -0.4 & -315.2 & -0.33 & -2.3 \\
${ }^{80} \mathrm{Sr}$ & -684.8 & 0.0 & +1.5 & -682.8 & 0.0 & +3.5 \\
${ }^{86} \mathrm{Sr}$ & -748.9 & 0.0 & 0.0 & -748.2 & 0.0 & +0.7 \\
${ }^{88} \mathrm{Sr}$ & -768.1 & 0.0 & +0.4 & -768.0 & 0.0 & +0.5 \\
${ }^{108} \mathrm{Mo}$ & -909.2 & -0.22 & +0.4 & -908.3 & -0.23 & +1.3 \\
${ }^{110} \mathrm{Mo}$ & -919.6 & -0.23 & -0.1 & -919.0 & -0.23 & +0.5 \\
${ }^{120} \mathrm{Xe}$ & -1008.2 & 0.26 & +0.3 & -1007.7 & 0.29 & +0.8 \\
${ }^{174} \mathrm{Yb}$ & -1407.2 & 0.31 & -0.6 & -1407.2 & 0.32 & -0.6 \\
\hline
\end{tabular}


It is noted that SIG-OM describes the experimental binding energies very well with only a few exceptions. The agreement is especially good for newly measured Si isotopes. Not surprisingly the force NL3 also shows reasonably good agreement for several data points. It, however, shows somewhat larger deviations from the data notably for the $\mathrm{Si}$ isotopes. Details of a comprehensive study to examine the shell structure and shell effects due to the Lagrangian model SIG-OM encompassing a larger number of data in the regions far away from the stability line will be discussed elsewhere [34].

\subsection{Breathing mode GMR energies and GCM calculations}

The incompressibility of nuclear matter $K$ is an important point on the equation of state (EOS) of nuclear matter. By definition, it is the curvature of the EOS at the saturation point. The value of $K$ is important as it affects the breathing mode giant monopole resonance (GMR) energies sensitively. As the force NL-SH has a rather large value, it is found to be not suitable for the GMR energies of nuclei. It may be recalled that within the $\sigma^{3}+\sigma^{4}$ model, the force NL3 was obtained with a value of incompressibility $K=271 \mathrm{MeV}$ which is in the range of physically acceptable values. In order to get a comparative picture of the response of various Lagrangians on the breathing-mode giant monopole resonance (GMR) energies, we have performed constrained generator coordinate method $(\mathrm{GCM})$ calculations for the GMR mode [35]. The nuclei included are ${ }^{90} \mathrm{Zr},{ }^{120} \mathrm{Sn}$ and ${ }^{208} \mathrm{~Pb}$. Experimental GMR energies on these nuclei are known with a reasonable precision [36 37]. The results of our calculations are shown in Table 6 and are compared with the experimental data. Clearly, the force NL-SH $(K=355 \mathrm{MeV})$ provides larger GMR energies due to its high incompressibility. NL3 $(K=271 \mathrm{MeV})$, on the other hand, underestimates the GMR data by $\sim 1 \mathrm{MeV}$. Results of our calculations obtained with NL3 agree well with those provided in ref. [7].

Table 5

The breathing mode GMR energies in nuclei obtained with the the constrained GCM calculations. The experimental data are from refs. [36 37]

\begin{tabular}{ccccc}
\hline Nucleus & SIG-OM & NL3 & NL-SH & exp. \\
\hline${ }^{90} \mathrm{Zr}$ & 18.2 & 16.9 & 19.5 & $17.81 \pm 0.30$ \\
${ }^{120} \mathrm{Sn}$ & 16.2 & 15.0 & 16.7 & $15.52 \pm 0.15$ \\
${ }^{208} \mathrm{~Pb}$ & 14.1 & 13.0 & 15.0 & $13.96 \pm 0.28$ \\
\hline
\end{tabular}

It is interesting to note that SIG-OM with its slightly lower value of $K=265$ 
MeV than that of NL3 provides GMR energies which are closer to the experimental data, especially those of ${ }^{90} \mathrm{Zr}$ and ${ }^{208} \mathrm{~Pb}$ considered frequently in analyses. In comparison, NL3 with a higher value of $K=271 \mathrm{MeV}$ underestimates the data by $\sim 1 \mathrm{MeV}$. Generally, a force with a lower value of $K$ would yield smaller values of GMR energies. This difference in the predictions of SIG-OM and NL3 may be due to a difference in some finite size effect of a nucleus. The other two factors which play a role for GMR energies are the Coulomb term and the asymmetry component. At present, it is difficult to estimate as to which factor brings about the apparent paradoxical behaviour of SIG-OM and NL3. We believe that it may be due to a different surface component (arising out of a different density dependence of the interactions) in SIG-OM vis-a-vis NL3. It will, therefore, be important to carry out calculations of surface incompressibility using the semi-infinite nuclear matter. These calculations are in progress. Moreover, it is also to be seen as to what response the two Lagrangian models provide in relativistic radom-phase approximation calculations of the breathing-mode GMR energies.

\section{$4.6 \quad$ Nuclear matter properties}

\subsubsection{Effective mass}

The density dependence of the effective mass $m^{*}$ is to an extent a reflection of the density dependence of interactions in a nucleus, though it corresponds to that of the $\sigma$ field to be exact. It would be instructive to see as to how $m^{*}$ changes with density in different Lagrangian models. We show in Fig. 3 the density dependence of $m^{*}$ for different Lagrangian models. Fig. 3(a) shows the effective mass $m^{*}$ for the density region of a nucleus up until a 4-5 times the saturation density. Both NL-SH and NL3 bunch out together and show a small difference in the high density region. NL-SV1 splits out from NL-SH and NL3 at higher densities. In the high-density region, three forces NL-SH and NL3 with the scalar self-couplings and NL-SV1 with the scalar self-coupling along with the vector self-coupling exhibit a similar pattern of variation with density as seen in Fig. 3(a). The model SIG-OM with $\sigma-\omega$ coupling, on the other hand, shows a density dependence that is different from that of NL-SH, NL3 and NL-SV1 and it is especially so in the high density region.

The region of the density relevant to finite nuclei is shown in Fig. 3(b). The saturation point is indicated by a square in each case. Both SIG-OM and NLSV1 have a slightly larger $m^{*}$ than that of NL-SH and NL3. It may be pointed out though that in each case the spin orbit splitting of ${ }^{16} \mathrm{O}$ is reproduced well and yet overall fits to finite nuclei lead to slightly different effective mass for each model. This is no coincidence that within the $\sigma^{3}+\sigma^{4}$ Lagrangian model, the effective masses for NL-SH and NL3 are very close to each other. The 

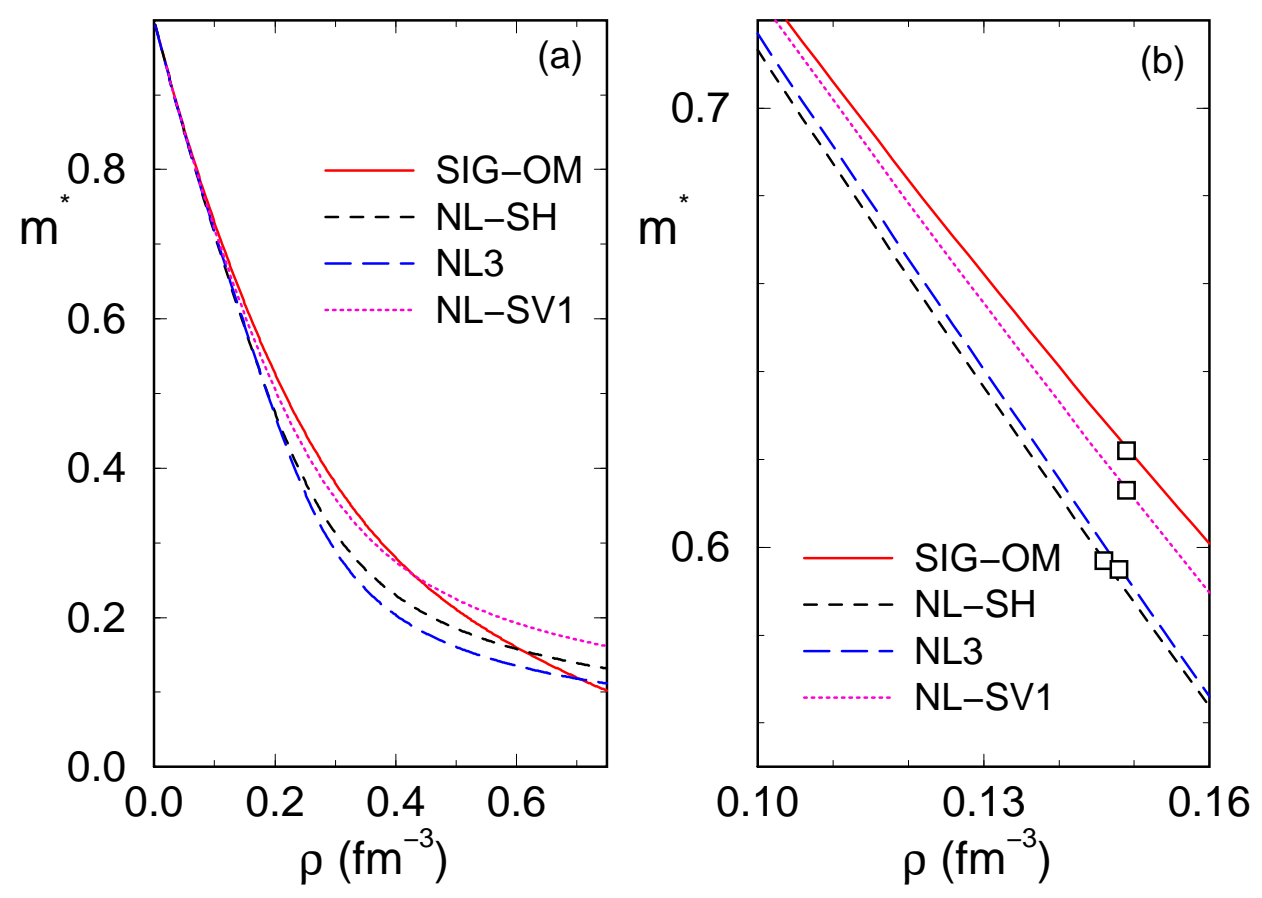

Fig. 3. The effective mass $m^{*}$ calculated with SIG-OM. It is compared with that for the $\sigma^{3}+\sigma^{4}$ Lagrangians NL-SH and NL3. A comparison is also made with that of $\omega^{4}$ Lagrangian NL-SV1. (a) The density dependence of $m^{*}$ for the whole density density including the high-density region. (b) $m^{*}$ in the region of density relevant to finite nuclei. The saturation point for each case is shown by squares.

variation in $m^{*}$ for these two forces in this region is also very similar. On the other hand, in the region of interest for finite nuclei, it is seen clearly that SIG-OM shows a density dependence that deviates strongly from that of NLSH and NL3. This may possibly lead to a different features of surface with SIG-OM. Detailed surface properties using various Lagrangian models are in progress. It is a matter for further investigation as to whether this difference in density dependence leads to different predictions of breathing-mode energies with SIG-OM as discussed above.

\subsubsection{The EOS of nuclear and neutron matter}

The EOS of nuclear and neutron matter is important for structure and properties of neutron stars. There has recently been a significant discussion on EOS of nuclear matter [38] especially in view of recent observations of neutron stars which have been found to exist with masses in the vicinity of two solar masses or even more [39]. In accordance with the known spectrum of neutron star masses which lie below $\sim 1.8$ solar mass $\left(M_{\odot}\right)$, the conventional wisdom has been that a softer EOS is required for describing the observed masses. However, recent discoveries of masses close to $2 M_{\odot}$ or more than $2 M_{\odot}$ seem to put new demands on the high-density EOS of nuclear matter. In ref. [39], the 
neutron star EXO 0748-676 with mass $M \geq 2.10 \pm 0.28 M_{\odot}$ has been reported. Consequently, very soft EOS for nuclear and neutron matter are ruled out. Then, one requires an EOS that should encompass even the heavier neutron star masses in the region of $2.0-2.3 M_{\odot}$.

Solving the equations for nuclear matter self-consistently, we have calculated the EOS of nuclear and neutron matter with the new Lagrangian model. The EOS of nuclear matter with SIG-OM shows a significant softening at higher densities as compared to that of NL-SH and NL3 (see Fig. 4). The coupling of $\sigma$ and $\omega$ meson does have a softening effect at higher densities. In contrast, EOS with the $\sigma^{3}+\sigma^{4}$ model is known to be stiff due to preponderance of $\omega$ term at higher densities.

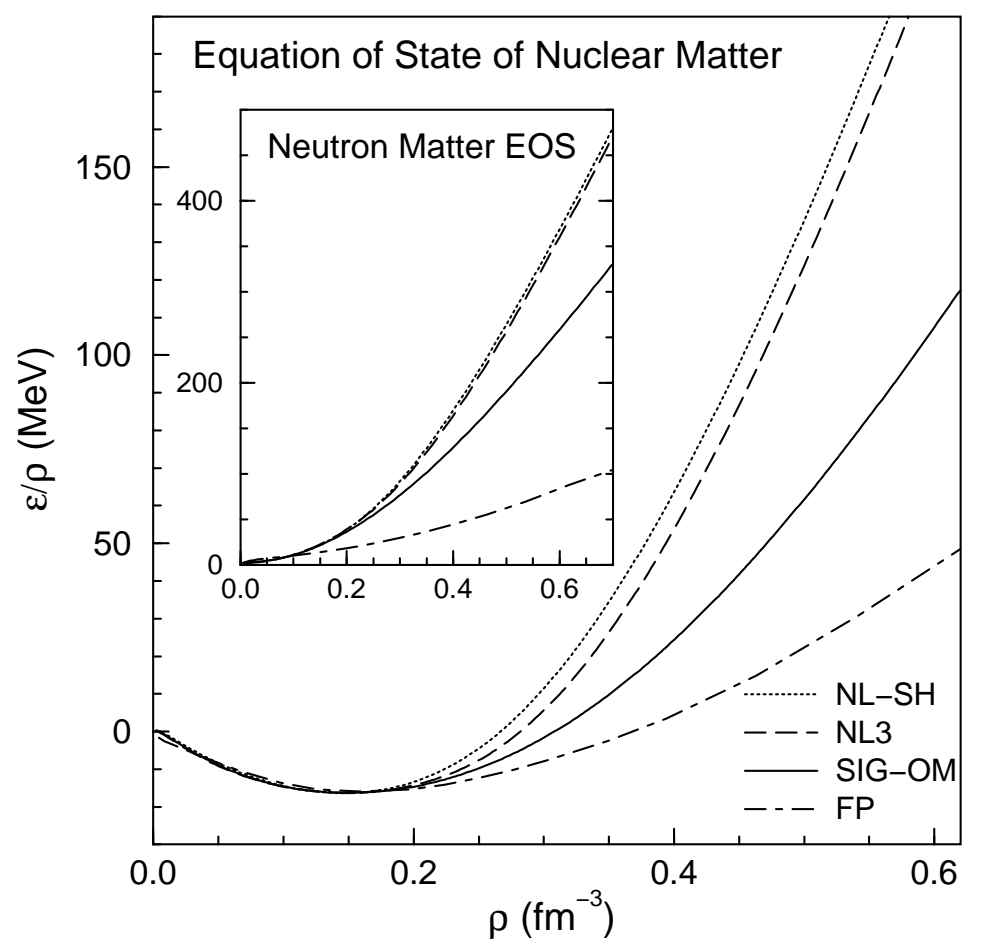

Fig. 4. The EOS of nuclear matter and neutron matter (inset) obtained with SIG-OM, NL-SH and NL3. A comparison is made with the EOS due to Friedman and Pandharipande (FP) [40].

The corresponding EOS for neutron matter is shown in the inset of Fig. 4. It can be seen that neutron EOS due to SIG-OM is also softer than that for NL-SH and NL3. For a comparison, we show the EOS due to Friedman and Padharipande (FP) [40]. The FP EOS for nuclear as well as neutron matter is very soft and was intended to describe lower mass neutron stars with masses $M<1.6 M \odot$. As one can see from Fig. 4, the softening achieved by the $\sigma-\omega$ coupling is not strong enough to match that of FP which is a very soft EOS leading to smaller values of maximum mass of neutron stars [40]. In view of 
new observations such as those of ref. [39], a softer EOS in the high density region may not be required. It has to be seen from neutron-star structure calculations whether the EOS due to SIG-OM which is neither as stiff as NL3 nor as soft as FP would be suitable for describing the newly observed neutron star masses.

\section{Conclusions and outlook}

The Lagrangian SIG-OM with the coupling of $\sigma$ and $\omega$ mesons in the RMF theory has been developed. We have explored the feasibility of adding the $\sigma-\omega$ coupling in the RMF Lagrangian. Properties of finite nuclei and nuclear matter have been explored with the model SIG-OM. It is shown that SIG-OM provides a very good description of the ground-state properties such as binding energies and charge radii of nuclei over a large range of isospin. Taking the case of the isotopic chain of Sn nuclei, it is shown that it describes the binding energies of nuclei between the two magic numbers $N=50$ and $N=82$ very well. It will be interesting to see in further investigations as to what implications this improvement in shell structural aspects may have in the other regions and most importantly in the regions close to the r-process path.

The breathing-mode giant monopole resonance energies for a few nuclei have been calculated using the constrained GCM calculations. It is shown that the model SIG-OM with the incompressibility of nuclear matter $K=265 \mathrm{MeV}$ describes the breathing-mode GMR energies well. A comparative analysis of breathing-mode GMR energies with the GCM approach in the RMF theory suggests that some finite-size effects with SIG-OM may be different than with Lagrangians with nonlinear scalar self-couplings. It is important to carry out calculations with relativistic RPA approach to see as to whether such a feature would persist. This is a matter of further investigations and requires a detailed study.

The equation of state of nuclear and neutron matter have been obtained with SIG-OM. A comparison is made with the EOS obtained with the interactions within the nonlinear scalar self-coupling. As it is well-known, the latter model gives very stiff EOS. The equation of state of nuclear and neutron matter

obtained with the new Lagrangian SIG-OM is significantly softer than that with non-linear scalar self-couplings.

We thank Prof. Lev Savushkin for fruitful discussions. 


\section{References}

[1] B.D. Serot and J.D. Walecka, Adv. Nucl. Phys. 16 (1986) 1.

[2] P.G. Reinhard, Rep. Prog. Phys. 52 (1989) 439.

[3] B.D. Serot, Rep. Prog. Phys. 55 (1992) 1855.

[4] P. Ring, Prog. Part. Nucl. Phys. 37 (1996) 193.

[5] Y.K. Gambhir, P. Ring, and A. Thimet, Ann. Phys. (N.Y.) 198 (1990) 132.

[6] M.M. Sharma, M.A. Nagarajan and P. Ring, Phys. Lett. B 312 (1993) 377.

[7] G.A. Lalazissis, J. König, and P. Ring, Phys. Rev. C 55 (1997) 540.

[8] M.M. Sharma, G.A. Lalazissis, and P. Ring, Phys. Lett. B 317 (1993) 9.

[9] M.M. Sharma, G.A. Lalazissis, J. König, and P. Ring, Phys. Rev. Lett. 74 (1994) 3744 .

[10] N. Tajima, P. Bonche, H. Flocard, P.-H. Heenen and M.S. Weiss, Nucl. Phys. A551 (1993) 434.

[11] P.-G. Reinhard and H. Flocard, Nucl. Phys. A584 (1995) 467.

[12] J. Boguta and A.R. Bodmer, Nucl. Phys. A292 (1977) 413.

[13] M.M. Sharma, A.R. Farhan and S. Mythili, Phys. Rev. C 61 (2000) 054306.

[14] Y. Sugahara and H. Toki, Nucl. Phys. A579 (1994) 557.

[15] H. Toki, H. Shen, K. Suniyoshi, H. Sugahara and I. Tanihata, J. Phys. G: Nucl. Part. Phys. 24 (1998) 1479.

[16] B.A. Nikolaus, T. Hoch, and D.G. Madland, Phys. Rev. C 46 (1992) 1757.

[17] J.J. Rusnak and R.J. Furnstahl, Nucl. Phys. A626 (1997) 495.

[18] T. Bürvenich, D.G. Madland, J.A. Maruhn, and P.G. Reinhard, Phys. Rev. C 65 (2002) 044308.

[19] R. Brockmann and H. Toki, Phys. Rev. Lett. 68 (1992) 3408.

[20] S. Typel and H.H. Wolter, Nucl. Phys. A656 (1999) 331.

[21] S. Typel, Phys. Rev. C 71 (2005) 064301.

[22] T. Niksic, D. Vretenar, P. Finelli and P. Ring, Phys. Rev. C 66 (2002) 024306.

[23] W. Long, J. Meng, N. Van Giai and S.G. Zhou, Phys. Rev. C 69 (2004) 034319.

[24] L.N. Savushkin et al, Phys. Rev. C 55 (1997) 167.

[25] R.J. Furnstahl, B.D. Serot, and H.B. Tang, Nucl. Phys. A 615 (1997) 441. 
[26] B.D. Serot, in Lecture Notes in Physics Vol. 641, (Springer Verlag, 2004) p. 31.

[27] B.G. Todd-Rutel, J. Pieckarewicz, Phys. Rev. Lett. 95 (2005) 122501.

[28] G. Audi and A.H. Wapstra, Nucl. Phys. A595 (1995) 409.

[29] J.F. Berger, M. Girod, and D. Gogny, Nucl. Phys. A428 (1984) 32.

[30] E.W. Otten, in Treatise on Heavy-Ion Science, edited by D.A. Bromley (Plenum, New York, 1989) Vol 7, p. 517.

[31] B. Jurado et al., Phys. Lett. B 649 (2007) 43.

[32] G. Sikler et al., Nucl. Phys. A763 (2005) 45.

[33] U. Hager et al., Phys. Rev. Lett. 96 (2006) 122501.

[34] M.M. Sharma, (unpublished, 2008)

[35] M.V. Stoitsov, P. Ring and M.M. Sharma, Phys. Rev. C50 (1994) 1445.

[36] D.H. Youngblood, H.L.Clark, Y.W. Lui, Phys. Rev. C69 (2004) 034315.

[37] M.M. Sharma et al., Phys. Rev C38 (1988) 2562.

[38] T. Klähn et al., Phys. Rev C74 (2006) 035802.

[39] F. Özel, Nature 441 (2006) 1115.

[40] B. Friedman and V.R. Pandharipande, Nucl. Phys. A361 (1981) 502. 